\title{
Cannabis and male reproduction: Impact on offspring via sperm epigenome
}

\author{
PAOLA GRIMALDI ${ }^{*}$ \\ Department of Biomedicine and Prevention, University of Rome Tor Vergata, Rome, 00133, Italy
}

Key words: Cannabis, Endocannabinoid system, Sperm, Paternal inheritance

\begin{abstract}
Cannabis is the most widely used drug in the Western societies particularly among adolescent and young adults. Epidemiological studies demonstrate that men use cannabis more frequently than women with higher risk in developing cannabis-related disorders. Although the direct adverse effects of cannabis on male reproductive functions have been studied both in humans and animal models, the possible long-term risks for the health of the users and their offspring are still an area of active research.
\end{abstract}

\section{Introduction}

Cannabis is the most widely used illicit drug in Western societies. In the last decade, with expanding cannabis legalization, its use strongly increased for medical and recreational purposes. It is estimated that $3 \%$ of the world's population consumes marijuana with an increase in the number of US cannabis users by 60\% between 2007 and 2017, mainly among males of reproductive age (World Drug Report, 2019). This trend is associated with a decrease in the perception of risk of harm of cannabis products and with an increase of its safety (Jordan et al., 2020). In this context, a change in the cannabis market has developed, leading to the availability of new forms of cannabis with a high content of tetrahydrocannabinol (THC), the main psychoactive component. In the early 1990s, the average THC content in marijuana was less than $4 \%$ while in 2018 it was more than 15\% (ElSohly et al., 2016; Stuyt, 2018). Although THC is the best-known psychoactive constituent of the plant, cannabis also contains more than 500 other chemicals, including more than 100 cannabinoids that are chemically related to THC but whose properties are still unknow (Amin and Ali, 2019). Higher potency strains of cannabis are associated with increased risk of developing a cannabis use disorder like psychosis and addiction (Blanco et al., 2016) and with a rise of concerns on the potential reproductive consequences, particularly in young males that are the most common users. Surprisingly, coincident are the observations of worldwide decline of semen quality in

*Address correspondence to: Paola Grimaldi, p.grimaldi@med.uniroma2.it Received: 22 May 2021; Accepted: 04 August 2021 developed countries and delayed conception for many couples compared to previous generations (Mann et al., 2020). Therefore, a possible link between use of cannabis and decline in sperm parameters and quality needs to be elucidated. Here, the current knowledges related to the adverse effects of $\mathrm{THC} /$ cannabinoids on male reproductive health are discussed. Special attention is paid to epigenetic modifications induced by the drug and potential risks of defects in the offspring transmitted by the sperm.

\section{Effect of cannabis on sperm parameters}

Both THC and cannabinoids exert their effects by binding to cannabinoid receptor type $1\left(\mathrm{CB}_{1}\right)$ and/or type $2\left(\mathrm{CB}_{2}\right) \mathrm{G}$ protein-coupled receptors, which are also the receptors of endogenous endocannabinoids (eCBs) like anandamide (AEA) and 2-arachidonoylglycerol (2-AG). All together cannabinoid receptors, eCBs and all the enzymes for the biosynthesis and degradation of eCBs, form the endocannabinoid system (ECS). The ECS is deeply involved in a broad array of biological functions and any interference with this system, through exogenous cannabinoids exposure, can alter the balance of downstream endogenous signaling pathways with possible pathological consequences (Maccarrone et al., 2015).

In male, the ECS is a key player in several reproductive processes both in humans and animals. In mouse, ECS is present and active in male germ cells and controls at least two critical steps of the germ cell differentiation: 1) the transition from mitotic to meiotic stage, during which testicular endocannabinoid 2-AG, produced by spermatogonia, might act as an autocrine factor via $\mathrm{CB}_{2}$ receptors promoting meiotic entry (Grimaldi et al., 2009); 2) spermiogenesis, during which AEA, produced by Sertoli cells and/or by spermatids, might 
act as a paracrine/autocrine factor via $\mathrm{CB}_{1}$ receptors to regulate sperm morphogenesis (Migliaccio et al., 2018). Since a similar expression pattern of many components of the ECS has been recently described in human male germ cells, ECS might play a similar role in human spermatogenesis (Nielsen et al., 2019). Accordingly, exposure to exogenous cannabinoid compounds that bind to $\mathrm{CB}_{1}$ and $\mathrm{CB}_{2}$ receptors could alter these critical steps of spermatogenesis, leading to altered sperm functions.

Several studies reported that cannabis consumption affects spermatogenesis and sperm functions, including sperm number, morphology, motility and lastly sperm epigenome (Grimaldi et al., 2013; Rossato et al., 2005; Barchi et al., 2020; Szutorisz and Hurd, 2016; Meccariello et al., 2020; Innocenzi et al., 2019). This last point is of particular significance since sperm epigenome modifications in cannabis consumers could have potential long-term effects on offspring health.

Both animal and in vitro studies reported a negative impact of cannabis (THC/cannabinoids) on male fertility with a reduction in sperm count and motility and with altered morphology (Murphy et al., 2018; Innocenzi et al., 2019; Whan et al., 2006; Verhaeghe et al., 2020). In humans, a few studies assessed the effects of cannabis smoking on male reproductive functions, reporting conflicting results. Gundersen et al. (2015) found that healthy young men who regularly smoked marijuana more than once per week had significantly lower sperm count than those who did not smoke marijuana or those who used the drug less frequently (Gundersen et al., 2015). In contrast, recently Nassan et al. (2019) reported that men that have smoked marijuana at some point in their life had significantly higher concentrations of sperm when compared with men who have never smoked marijuana. One possible hypothesis has been suggested: a moderate use of marijuana may have a pro-spermatogenic effect improving testicular function while at higher dose, this relation reverses, resulting in adverse effects (Nassan et al., 2019). However, this aspect, in humans, remains a matter of debate and requires further investigation.

\section{Effect of cannabis on sperm epigenome}

One of the most exciting area of research is the effect of cannabinoid/THC on sperm epigenome that aims to identify the epigenetic modifications induced by the drug in the sperm (Schrott and Murphy, 2020). The establishment and maintenance of the paternal epigenetic program is important for appropriate gamete function and quality and alterations of sperm epigenome may influence the health and development of next generations. Epigenetic mechanisms include DNA methylation at CG dinucleotides, post-translational modifications of histones tails, and noncoding RNA (ncRNA). These modifications regulate gene expression without altering the DNA sequence and are important modulator of cell differentiation, including spermatogenesis. In animal models several evidence showed that paternal exposure to $\mathrm{THC} / \mathrm{cannabinoid} \mathrm{has} \mathrm{effects} \mathrm{on}$ health of next generation, causing behavioural and neurobiological abnormalities in the offspring. However, to date, only few evidence in animal models have linked epigenetic modifications identified in the offspring with those present in the sperm of THC/cannabinoids exposed animals.

In a study, Murphy et al. (2018) reported that cannabis use in humans and THC exposure in rats, is associated with widespread changes of sperm DNA methylation (Murphy et al., 2018). In a follow up study, they found that sperm of rat exposed to Cannabis showed hypomethylation in the autism candidate gene Dlgap2 and that the same hypomethylated state of this gene was present in the nucleus accumbens of rat brain born from THC exposed fathers, strongly supporting the potential inheritance of an altered sperm DNA methylation pattern (Schrott et al., 2019). Besides Dlgap2, the same group identified other genes involved in neurodevelopment that are differentially methylated in sperm of THC exposed rats respect to control unexposed rats, suggesting that could be inherited by the offspring determining aberrant expression in the brain (Levin et al., 2019; Schrott et al., 2020).

In a study performed by our group, the effect of the synthetic cannabinoid JWH-133, selective agonist of $\mathrm{CB}_{2}$ receptor, on mouse spermatogenesis and sperm quality has been investigated (Grimaldi et al., 2009; di Giacomo et al., 2016). The interest towards this receptor is based on its high level of expression in spermatogonia. In in vitro experiments, JWH-133 treatment of spermatogonia induced changes in histone methylation levels of $\mathrm{H} 3 \mathrm{~K} 4 \mathrm{~m} 3$ and $\mathrm{H} 3 \mathrm{~K} 9 \mathrm{~m} 2$ in genomic flanking regions of meiotic genes $c$-Kit and Stra8, increasing their expression and promoting meiotic entry of these cells (di Giacomo et al., 2016). As consequence, JWH-133 chronic exposure of immature male mice showed accelerated spermatogenesis onset and reduced spermatozoa number recovered by epididymis, pointing out the importance of correct endocannabinoid signaling for proper spermatogenesis (di Giacomo et al., 2016). Furthermore, in a follow up study we reported that sperm from JWH-133 treated mice maintained the ability to fertilize egg, but embryo growth and placenta development were impaired. One possible explanation is that in vivo over-activation of this receptor, through JWH-133 exposure of male mice, could determine epigenetic aberrations in germ cells that are then paternally inherited by the offspring through the sperm. We demonstrated that DNA methylation of paternally expressed imprinted genes Peg10 and Plagl1, two genes important for placental development, resulted hypermethylated in sperm of JWH-133 exposed male and the same DNA modification was retained in the placenta and associated with reduced placenta development and embryo growth in the progeny from JWH-133 exposed father (Innocenzi et al., 2019). These is the first evidence of epigenetic changes induced by $\mathrm{THC} /$ cannabinoids in germ cells and transmitted to the new individual by sperm. A proposed model is reported in Fig. 1.

\section{Conclusions and perspectives}

In the recent years increasing evidence indicate that father's lifetime experiences, such as cannabis use, can be transmitted to the offspring, affecting health and development. Thus, it is important to know the potential consequences of cannabis exposure not only in the father but also in the offspring. Recent studies suggested that 


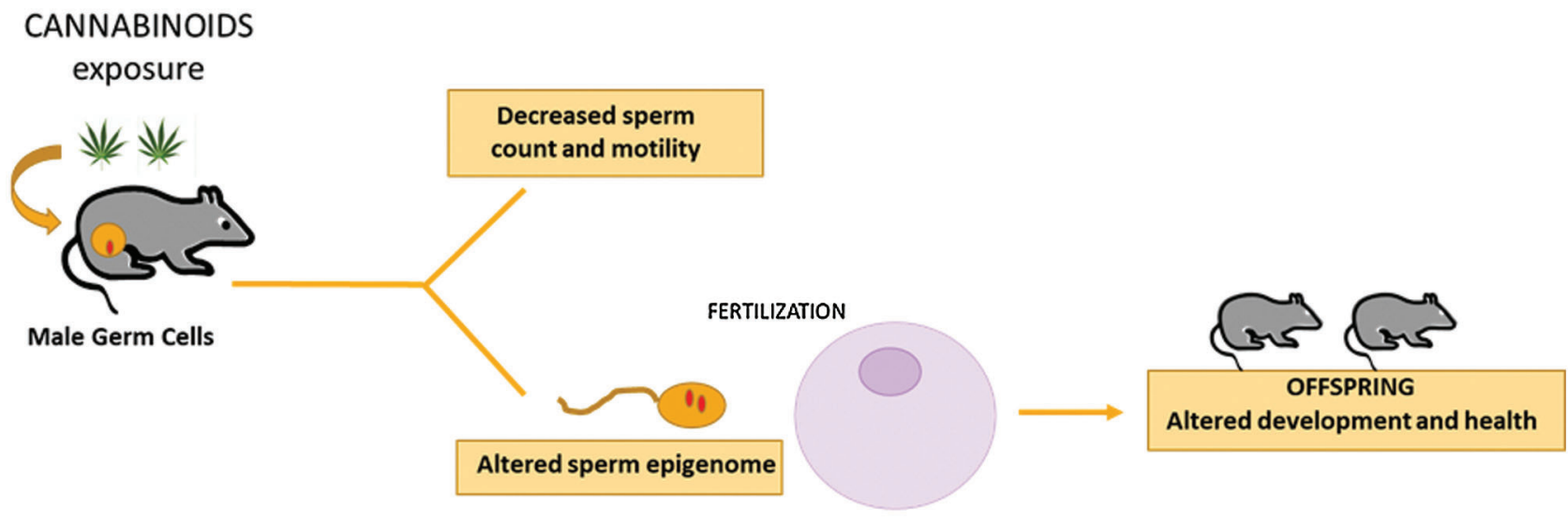

Epigenetic modifications

FIGURE 1. Cannabinoid exposure on male reproduction. Cannabinoid exposure affects male reproduction by impairing sperm number and motility in the exposed animal and by altering development and health of the offspring via sperm epigenome.

epigenetic alterations in the sperm could be involved in the transmission of cannabis-induced traits from father to offspring. Therefore, more attention needs to be paid to fathers' lifestyle and on the potential effects on sperm epigenome. Prevention should encourage men to live healthy lives and should increase risks perception of cannabis consumption to protect the health of users and their offspring.

Availability of Data and Materials: No data are included within this Viewpoint.

Authors' Contribution: The author confirms sole responsibility for the following: study conception and design and manuscript preparation.

Funding Statement: The author received no specific funding for this study.

Conflicts of Interest: The author declares that she has no conflicts of interest to report regarding the present study.

\section{References}

Amin MR, Ali DW (2019). Pharmacology of medical cannabis. Advances in Experimental Medicine and Biology 1162: 151-165.

Barchi M, Innocenzi E, Giannattasio T, Dolci S, Rossi P et al. (2020). Cannabinoid receptors signaling in the development, epigenetics, and tumours of male germ cells. International Journal Molecular Science 21: 25.

Blanco C, Hasin DS, Wall MM, Florez-Salamanca L, Hoertel N et al. (2016). Cannabis use and risk of psychiatric disorders: prospective evidence from a US national longitudinal study. JAMA Psychiatry 73: 388-395.

di Giacomo D, de Domenico E, Sette C, Geremia R, Grimaldi P (2016). Type-2 cannabinoid receptor contributes to the physiological regulation of spermatogenesis. FASEB Journal 30: 1453-1463.

ElSohly MA, Mehmedic Z, Foster S, Gon C, Chandra S, Church JC (2016). Changes in cannabis potency over the last 2 decades (1995-2014): Analysis of current data in the United States. Biological Psychiatry 79: 613-619.

Grimaldi P, Di Giacomo D, Geremia R (2013). The endocannabinoid system and spermatogenesis. Frontiers in Endocrinology (Lausanne) 16: 192.
Grimaldi P, Orlando P, Di Siena S, Lolicato F, Petrosino S et al. (2009). The endocannabinoid system and pivotal role of the CB2 receptor in mouse spermatogenesis. Proceedings of the National Academy of Sciences of the United States of America 106: 11131-11136.

Gundersen TD, Jørgensen N, Andersson AM, Bang A, Nordkap AK et al. (2015). Association between use of marijuana and male reproductive hormones and semen quality: A study among 1,215 healthy young men. American Journal of Epidemiology 182: 473-481.

Innocenzi E, De Domenico E, Ciccarone F, Zampieri M, Rossi G, Cicconi R, Bernardini R, Mattei M, Grimaldi P (2019). Paternal activation of $\mathrm{CB} 2$ cannabinoid receptor impairs placental and embryonic growth via an epigenetic mechanism. Scientific Reports 9: 17034.

Jordan T, Ngo B, Jones CA (2020). The use of cannabis and perceptions of its effect on fertility among infertility patients. Human Reproduction Open 2020: 1-8.

Levin ED, Hawkey AB, Hall BJ, Cauley M, Slade S et al. (2019). Paternal THC exposure in rats causes long-lasting neurobehavioral effects in the offspring. Neurotoxicology and Teratology 74: 106806.

Maccarrone M, Bab I, Bíró T, Cabral GA, Dey SK et al. (2015). Endocannabinoid signaling at the periphery: 50 years after THC. Trends in Pharmacological Sciences 36: 277-296.

Mann U, Shiff B, Patel P (2020). Reasons for worldwide decline in male fertility. Current Opinion in Urology 30: 296-301.

Meccariello R, Santoro A, D'Angelo S, Morrone R, Fasano S, Viggiano A, Pierantoni R (2020). The epigenetics of the endocannabinoid system. International Journal of Molecular Sciences 21: 1113.

Migliaccio M, Ricci G, Suglia A, Manfrevola F, Mackie K et al. (2018). Analysis of endocannabinoid system in rat testis during the first spermatogenetic wave. Frontiers in Endocrinology 9: 269.

Murphy SK, Itchon-Ramos N, Visco Z, Huang Z, Grenier C et al. (2018). Cannabinoid exposure and altered DNA methylation in rat and human sperm. Epigenetics 13: 1208-1221.

Nassan FL, Arvizu M, Mínguez-Alarcón L, Williams PL, Attaman J et al. (2019). Marijuana smoking and markers of testicular function among men from a fertility centre. Human Reproduction 34: 715-723.

Nielsen JE, Rolland AD, Rajpert-De Meyts E, Janfelt C, Jørgensen A et al. (2019). Characterisation and localisation of the 
endocannabinoid system components in the adult human testis. Scientific Reports 9: 12866.

Rossato M, Popa FI, Ferigo M, Clari G, Foresta C (2005). Human sperm express cannabinoid receptor CNR1, the activation of which inhibits motility, acrosome reaction and mitochondrial function. Journal of Clinical Endocrinology and Metabolism 90: 984-991.

Schrott R, Acharya K, Itchon-Ramos N, Hawkey AB, Pippen E et al. (2019). Cannabis use is associated with potentially heritable widespread changes in autism candidate gene DLGAP2 DNA methylation in sperm. Epigenetics 15: 161-173.

Schrott R, Murphy SK (2020). Cannabis use and the sperm epigenome: A budding concern? Environmental Epigenetics 6: 1-10.

Schrott R, Rajavel M, Acharya K, Huang Z, Acharya C et al. (2020). Sperm DNA methylation altered by THC and nicotine:
Vulnerability of neurodevelopmental genes with bivalent chromatin. Scientific Reports 10: 16022.

Stuyt E (2018). The problem with the current high potency THC marijuana from the perspective of an addiction psychiatrist. Missouri Medicine 115: 482-486.

Szutorisz H, Hurd YL (2016). Epigenetic effects of cannabis exposure. Biological Psychiatry 79: 586-594.

World Drug Report (2019). UNODC.

Verhaeghe F, Di Pizio P, Bichara C, Berby B, Rives A et al. (2020). Cannabis consumption might exert deleterious effects on sperm nuclear quality in infertile men. Reproductive Biomedicine Online 40: 270-280.

Whan LB, West MC, McClure N, Lewis SE (2006). Effects of delta-9tetrahydrocannabinol, the primary psychoactive cannabinoid in marijuana, on human sperm function in vitro. Fertility and Sterility 85: 653-660. 